\title{
Selective attention in the speeded classification and comparison of multidimensional stimuli
}

\author{
JEFFREY L. SANTEE and HOWARD E. EGETH \\ The Johns Hopkins University, Baltimore, Maryland 21218
}

\begin{abstract}
Selective attention was studied when subjects were required to make either speeded classifications of single stimuli or comparisons of pairs of multidimensional stimuli. Experiment 1 established that subjects were able to attend selectively to form when the irrelevant dimension of size or shading varied in a speeded-classification (card-sorting) task. Experiment 2 confirmed this finding in a discrete-trials task. However, subjects were not able to filter out irrelevant dimensional disparity in a comparably designed simultaneous-comparison (i.e., "same"-"different") task. Mean "same" reaction time increased monotonically with increases in disparity between the two stimuli on the irrelevant dimension. Experiment 3 also revealed a monotonic increase in "same" RT as a function of irrelevant disparity in a successive-comparison task. These results were discussed in terms of a normalization model proposed by Dixon and Just (1978) in which it is assumed that a subject equates the two stimuli on the irrelevant dimension before deciding that they are the same along the relevant dimension. It was concluded that: (1) although subjects can efficiently filter out irrelevant disparity in a speeded-classification task, interference due to irrelevant disparity is obtained in the comparison tasks, (2) a common process such as normalization does not necessarily underlie performance in the speeded-classification and comparison tasks, (3) the ability to attend selectively to a stimulus dimension may be task determined as well as stimulus determined, and (4) contrary to the Dixon and Just proposal, normalization of irrelevant disparity occurs in a comparison task, even when the relevant dimension is represented as a separate encoding feature.
\end{abstract}

In recent years, considerable attention has been devoted to understanding how multidimensional stimuli are perceived. Typically, multidimensional stimuli are generated from two or more dimensions that are dichotomous and orthogonal to one another (see Garner, 1974b, for a detailed review). The stimuli are then presented within the context of one of the major paradigms described below.

\section{Speeded Classification}

In the speeded-classification task, popularized by Garner and his colleagues, stimuli are presented one at a time, and the time required to classify test stimuli according to an experimenter-determined stimulus dimension is measured (e.g., Garner, 1974a, 1974b, 1976, 1978; Garner \& Felfoldy, 1970; Gottwald \& Garner, 1972, 1975; Pomerantz \& Garner, 1973). The speed of classification is usually measured in three conditions: (1) when the test stimuli vary on a single dimension, (2) when they differ on correlated or redundant stimulus dimensions, and (3) when the

This research was supported in part by a grant from the National Science Foundation (BNS-76-01227) to Howard Egeth and James Pomerantz. The authors would like to thank Peter Dixon, Lester Krueger, Michael McCloskey, and James Pomerantz for their helpful advice and Lori Plotkin for assistance in running subjects. Requests for reprints should be sent to either Jeffrey Santee or Howard Egeth, Department of Psychology, The Johns Hopkins University, Baltimore, Maryland 21218. stimuli vary on dimensions combined orthogonally. In each condition, the subject is instructed to classify the stimuli according to a single, specified dimension, referred to as the relevant dimension. Performance in the single-dimension condition is then used as a baseline measure to be compared with the speed of classification when the second (irrelevant) dimension is correlated with, or is orthogonal to, the relevant dimension. The orthogonal condition is also referred to as a filtering task because the subject attempts to filter out variation on the irrelevant dimension while attending to the relevant dimension (Posner, 1964). The ability to attend selectively to the relevant dimension is determined by the relative loss of sorting speed in the orthogonal condition compared with the single-dimension condition.

On the one hand, dimensions such as the height and width of rectangles (Felfoldy, 1974) and the Munsell color dimensions of value and chroma (Garner \& Felfoldy, 1970) produce a redundancy gain in sorting speed when the dimensions are correlated, and result in a significant loss in speed when the dimensions are varied orthogonally. This pattern of results defines integral dimensions. On the other hand, dimensions such as the size of a circle and the tilt of an inscribed line or the form and color of geometric figures (Gottwald \& Garner, 1972) do not produce a redundancy gain or orthogonal interference. This pattern of results defines separable dimensions. 
Thus, nearly perfect selective attention to one stimulus dimension is possible in the filtering task with separable, but not with integral, dimensions.

\section{Simultaneous Comparison}

Although selective attention to one dimension of a multidimensional stimulus has been shown to vary as a function of the particular dimensions used, a question that also deserves consideration is whether selective attention to a particular dimension is task dependent. More specifically, does variation on an irrelevant dimension affect performance differently when subjects are required to make a speeded classification of individually presented stimuli than when they are required to decide if two simultaneously presented stimuli are the "same" or "different"? A comparison between these two paradigms based on the existing literature suggests that subjects cannot or do not filter out irrelevant information as efficiently in the simultaneous-comparison task as in the speededclassification task. That is, reaction time (RT) in the simultaneous-comparison task increases when the irrelevant dimension is in a state that is incompatible with the relevant dimension (Egeth, 1966; Hawkins, McDonald, \& Cox, 1973; Hawkins \& Shigley, 1972; Keuss, 1977). It is important to note that the dimensions that produced interference in these simultaneouscomparison studies (color, form, size of a circle, and tilt of an inscribed line) are dimensions that failed to produce orthogonal interference in a speededclassification task.

The effects of irrelevant dimensional variation on simultaneous comparisons have not been restricted to the use of geometric stimuli. Krueger (1973), for example, reported that "same" judgments of letter pairs are lengthened when the letters are flanked asymmetrically by irrelevant symbols. Besner and Coltheart (1975) found that word pairs differing in size slowed "same" RTs compared with pairs of words composed of the same size font. In addition, Egeth and Gilmore (Note 1) reported that "same" judgments of a letter pair (e.g.,, $\mathcal{C}$ ) are faster when embedded in a word context (e.g., ACE) than when in a nonword context (e.g., VCH). It appears that subjects were unable to attend selectively to the middle letter position, despite the fact that they were instructed to do so. ${ }^{1}$ These and similar results have led some investigators to conclude that "same" judgments may be mediated by a holistic process that is responsive to the entire configuration of the stimulus array (e.g., Egeth \& Blecker, 1971; Krueger, 1973).

\section{Interference in Speeded Classification and Simultaneous Comparison}

Recently, Dixon and Just (1978) have suggested that the orthogonal interference between dimensions in speeded classification and the interference caused by disparity on the irrelevant dimension in simultaneous- comparison tasks may be mediated by a common underlying process. They contend that interference occurs in both tasks when two stimuli that vary on the irrelevant dimension are compared with one another. It is assumed that on each trial of a classification task, rather than basing a response solely on the stimulus presented, a subject compares the stimulus presented with his representation of the one presented on the preceding trial. If the two stimuli match, then the same response is given as was given on the previous trial. If the two stimuli differ in the single-dimension condition, then a different response must be selected, creating a cost in RT. Felfoldy (1974), using height and width of rectangles as his stimulus dimensions, has provided support for such an analysis of the speeded-classification task. He noted that there are more unique stimuli in an orthogonal condition than in a single-dimension condition. Consequently, there are fewer stimulus and response repetitions in the former case than in the latter. This, in turn, translates into longer RT (i.e., interference) in the orthogonal condition. Felfoldy also reported a significant amount of interference in the orthogonal condition when his stimuli, which were composed of integral dimensions, changed from one trial to the next along the irrelevant dimension even though the response remained the same. Thus, interference occurs in the speeded-classification task when there is disparity between successive stimuli on the irrelevant dimension. Similarly, interference occurs in the simultaneous-comparison task when there is disparity between the to-be-compared stimuli on the irrelevant dimension.

Dixon and Just (1978) propose that stimulus normalization is the common process that underlies interference in both tasks. Two stimuli, whether they are presented side by side in the simultaneouscomparison task or in a sequential manner in the speeded-classification task, must be "mentally equated" on the irrelevant dimension. Moreover, the greater the amount of irrelevant disparity, the more time is required for normalization, since normalization entails the continuous transformation of irrelevant disparity. ${ }^{2}$ Dixon and Just have supported their normalization hypothesis by showing that the time required for "same" judgments in a simultaneouscomparison task increases monotonically with the amount of disparity between two stimuli on the irrelevant dimension. The stimuli used in this demonstration were composed of integral dimensions: heights and widths of ellipses, and hues and tints of color patches.

Although the proposed explanation of interfirience effects in speeded-classification and simultaneouscomparison tasks is provocative, there are some possible problems with the explanation that suggest the need for further empirical work. Let's consider some of these potential problems. 
(1) The contention that speeded classification involves a comparison between successive stimuli is based on Felfoldy's (1974) study of stimulus and response repetition effects in which the response stimulus interval (RSI) ranged from 82 to $1,080 \mathrm{msec}$. We believe that the generality of Felfoldy's interpretation of orthogonal interference is limited in some respects. In the first place, of course, short RSIs do not always lead to interference between dimensions: separable dimensions such as color and form do not interfere with one another even with the extremely short RSIs of the card-sorting task. In the second place, even when it occurs, orthogonal interference is not necessarily a result of repetition effects. Dykes and Cooper (1978), in a relative coding task, used the same integral dimensions as Felfoldy and still obtained sizable interference effects without the aid of any intertrial stimulus repetitions.

(2) Felfoldy's data speak to the issue of interference in speeded classification. Dixon and Just's case would be stronger if it could also be shown that normalization occurs in the speeded-classification task. To our knowledge, no such effect has yet been demonstrated. [It is important to keep in mind that normalization is not the same as interference. To demonstrate normalization, it is necessary to: (a) have at least three values along a stimulus dimension and (b) obtain a monotonic relationship between a performance measure and the amount of disparity between stimuli.]

(3) If we assume for the moment, based on Dixon and Just's (1978) suggestion, that interference in the speeded-classification and simultaneous-comparison tasks is mediated by a common normalization process, then both tasks would be expected to exhibit (or fail to exhibit) interference for the same pairs of stimulus dimensions. Dixon and Just also suggest that normalization should not occur with separable dimensions in the simultaneous-comparison task, since subjects should be able to access the relevant dimension without referencing the irrelevant dimension. However, as noted earlier, separable dimensions such as color and form (Gottwald \& Garner, 1972), which fail to produce interference in a speededclassification task, have been shown to produce interference in a simultaneous-comparison task (e.g., Egeth, 1966; Hawkins \& Shigley, 1972). Whether such dimensions will produce normalization (as opposed simply to interference) is an open question. To our knowledge, a direct comparison between speeded-classification and simultaneous-comparison tasks while employing the same stimulus dimensions has not been made.

\section{Purpose of Present Studies}

Experiments 1 and 2 test whether two stimulus dimensions that fail to cause interference in a speededclassification task will result in interference in a simultaneous-comparison task. Experiment 3 examines whether interference in a comparison task is dependent on simultaneity of presentation by presenting stimuli in a successive-comparison paradigm. Dixon and Just's (1978) normalization hypothesis leads to the prediction that interference will not be obtained between separable dimensions in either comparison task, since the relevant dimension can be represented as a separate "encoding feature." In such a case, the two stimuli could be compared directly on the relevant dimension without perceptually referencing the irrelevant dimension. Consequently, a failure of selective attention in the comparison tasks would suggest that: (1) the basis for interference effects in the speeded-classification and comparison tasks may be different, (2) selective attention to a stimulus dimension can be task determined as well as stimulus determined, and (3) selective attention may filter out irrelevant disparity more efficiently in the speeded-classification paradigm.

\section{EXPERIMENT 1}

Experiment 1 was conducted to determine whether subjects can selectively attend to the form of a geometric figure in a speeded-classification task when the irrelevant dimension is either size or shading. Card-sorting times were obtained when only form varied and when form was varied orthogonally with either size or shading. Since we were not concerned with completely establishing the separability and integrality of these dimensions, neither the correlated dimensions condition nor classification with size and shading as the relevant dimensions were conducted. Size and shading were chosen as the irrelevant dimensions so that the amount of disparity on these dimensions could be varied in continuous steps.

\section{Method}

Subjects. Sixteen undergraduates at Johns Hopkins University served as paid subjects. The irrelevant dimension for eight of the subjects was size, whereas for the remaining eight subjects it was shading. Each subject was paid $\$ 3.00$ for participating in the 45-min experimental session.

Stimulus materials-Form and size. Two outline forms (circle, square) and three sizes (small, medium, large) were employed. Each stimulus was drawn on a $6.8 \times 8.8-\mathrm{cm}$ white card with a black fine-tipped pen. Each card was covered with a transparent plastic coating in order to facilitate sorting of the cards. Square and circle templates were used to generate the geometric figures. The sizes of the squares and diameters of the circles were .64, 1.27, and $2.54 \mathrm{~cm}$ for the small, medium, and large stimuli, respectively. Thus, the ratios of the small-to-medium-to-large figures were approximately 1:2:4. At a typical viewing distance of about $30 \mathrm{~cm}$, the small, medium, and large figures subtended visual angles of $1.2,2.4$, and $4.8 \mathrm{deg}$, respectively.

Stimulus materials-Form and shading. Two values of form (circle, square) and three levels of shading (light, medium, dark) were used. The sides of the squares and the diameters of the circles measured $2.54 \mathrm{~cm}$. Shading was varied by using three intensities of neutral gray shading film manufactured by Chartpak: $10 \%$ (light), $40 \%$ (medium), and $70 \%$ (dark). 
Each subject was assigned randomly to either a size-irrelevant or shading-irrelevant group. Within each experimental group, three single-dimension decks were sorted in which form varied while the irrelevant dimension remained at a constant level. Each deck corresponded to one of the three levels of the irrelevant dimension. An orthogonal deck was also used in which form and the irrelevant dimension varied independently. All decks contained 36 stimuli: 18 circles and 18 squares. The six different stimuli ( 2 forms by 3 sizes/shades) appeared equally often in the orthogonal deck.

Procedure. A subject was requested to sort each deck into two groups-one for circles and one for squares-as quickly and accurately as possible. Each trial began by handing the subject a shuffled deck with the cards faceup. The experimenter then gave a verbal "ready" signal, waited for the subject's acknowledgment, and then pressed down on a telegraph key which started a digital timer. The click of the key served as a "go" signal to the subject. As soon as the last card was laid down, the subject hit another nearby key in order to stop the clock. The experimenter then recorded the time and noted any errors that had been observed during the sort. No feedback was provided. Times were recorded to the nearest millisecond.

The order of the four decks (three single-dimension and one orthogonal deck) was arranged according to a 4 by 4 Latin square such that each subject received one particular order. Each of the four primary orders was repeated twice across the eight subjects in each group of the experiment. Each subject was run on six blocks of trials--three times on all decks in a particular order and three more times on all decks in the reverse order. The first two blocks served as practice, and no data from these two blocks were analyzed.

\section{Results and Discussion}

The data from the three single-dimension decks (tasks) were pooled and compared against performance in the orthogonal task. The mean sorting times for the size-irrelevant and shading-irrelevant groups were entered into separate 2 (tasks) by 4 (blocks) by 8 (subjects) repeated-measures analyses of variance. There were too few errors to warrant analysis.

Shown in Table 1 are the mean sorting times averaged across the four blocks of trials in the singledimension and orthogonal tasks when size and shading were irrelevant. Sorting times decreased $.6 \mathrm{sec}$ from Block $1(15.4 \mathrm{sec})$ to Block $4(14.8 \mathrm{sec})$ in the size group $[F(3,21)=3.41, p<.05]$ and decreased $.8 \mathrm{sec}$ in the shading group from Block $1(16.4 \mathrm{sec})$ to Block $4(15.6 \mathrm{sec})[\mathrm{F}(3,21)=6.69, \mathrm{p}<.005]$. These data indicate that our subjects became increasingly more proficient at making form classifications across the four experimental blocks. Blocks did

Table 1

Mean Sorting Times (in Seconds) and Frequency of Errors per Deck for Single-Dimiension and Orthogonal Tasks With the Irrelevant Dimensions of Size and Shading

\begin{tabular}{cccccc} 
& \multicolumn{3}{c}{ Irrelevant Dimension } \\
\cline { 2 - 5 } \multicolumn{1}{c}{ Task } & Time & Errors & & Time & Errors \\
\cline { 2 - 5 } & Timading & \\
Single Dimension & 15.06 & .10 & 16.02 & .13 \\
Orthogonal & 15.12 & .03 & 16.01 & .12 \\
\hline
\end{tabular}

not interact with tasks in either the size $(p>.40)$ or shading $(p>.20)$ group. Most importantly, no differences between the single-dimension and orthogonal tasks were obtained in the size $(p>.20)$ and shading $(p>.40)$ groups.

These results indicate that in a speeded-classification task, subjects are able to attend selectively to form when the irrelevant dimension of either size or shading varies.

\section{EXPERIMENT 2}

Experiment 1 established that subjects can attend selectively to form while using the kind of cardsorting task often used by Garner and his colleagues. Experiment 2 was designed to determine whether the filtering of irrelevant dimensional disparity varies as a function of the specific task employed. Discrete trials were used in Experiment 2 so that we might replicate the card-sorting results, analyze stimulus sequence effects, and compare our results with the results from comparable studies of selective attention. In the speeded-classification task, subjects were required to determine whether each individually presented stimulus was a circle or a square on each trial. The comparison task required subjects to determine whether the two simultaneously presented stimuli were the "same" or "different" with respect to form, regardless of any differences in size or shading. The two tasks were equated with respect to stimulus composition and repetitions, response probabilities, and subjects.

Since subjects can attend selectively to form when speeded classification requires cards to be sorted, the same pattern of results (i.e., no interference) might be expected in Experiment 2 when simultaneous comparison is required. However, a number of studies are available which suggest that physical variation in size or contrast can affect "same".-"different" comparisons. Bundesen and Larsen (1975; Experiment 2) found that both "same" and "different" judgments of the form of two simultaneously presented stimuli increased linearly as a function of the (irrelevant) size ratio of the pair of stimuli. That is, the larger the difference in size between the two forms, the longer it took for subjects to make either "same" or "different" judgments of form. Recently, these authors replicated this linear relationship while using a successive-comparison paradigm (Larsen \& Bundesen, 1978; Experiment 1). Besner and Coltheart (1976) also obtained a monotonic increase in "same" RT with increases in the magnitude of the relative size differences between stimulus pairs in both simultaneous- and successive-comparison tasks. Contrary to Bundesen and Larsen (1975), "different" RTs were not affected by relative size differences.

In these studies, the stimuli were either randomly generated closed figures (Bundesen \& Larsen, 1975; 
Larsen \& Bundesen, 1978) or open stick figures (Besner \& Coltheart, 1976) designed to avoid name associations that could be used to make "same""different" judgments. However, similar results have also been obtained with highly overlearned linguistic materials such as letters (Corcoran \& Besner, 1975) and words (Besner \& Coltheart, 1975). For example, Corcoran and Besner (1975) found that it takes approximately $25 \mathrm{msec}$ longer to make a "same name" response to the letter pair $\mathrm{BB}$ than to the pair BB. Only a $2: 1$ size ratio was used, so it is impossible to determine whether even greater increases in "same" RT would have resulted from larger relative size differences. Nevertheless, it remains clear that at least "same" RTs are longer when there is disparity on the irrelevant dimension of size for both figures and linguistic symbols. Corcoran and Besner (1975) have also shown that a difference in light-dark contrast between two otherwise physically identical letters increases "same name" RT. Thus, disparity on the irrelevant dimension of either size or shading was expected to produce interference in the simultaneous-comparison task.

Dixon and Just (1978) suggest that normalization occurs when integral dimensions are varied in simultaneous-comparison tasks. They argue that in order for normalization to occur, subjects must not be able to directly encode the levels of the relevant dimension. Otherwise, the relevant dimension will be accessed directly without perceptually referencing the irrelevant dimension. As noted earlier, evidence concerning normalization has been generated from stimuli that cannot be easily named or represented as an encoding feature. However, the interference that arises from size or contrast differences between two otherwise physically identical letters suggests that subjects tend to access a physical code even when a name code may be accessed independently (Corcoran \& Besner, 1975; Posner, 1978; Posner \& Mitchell, 1967).

Three levels of size and three levels of shading were used in this experiment in order to determine if normalization occurs even when the relevant dimension contains easily accessible encoding features such as the names of common geometric figures.

\section{Method}

Subjects. Twelve undergraduates at Johns Hopkins University served as paid subjects. Each had normal or corrected-to-normal vision.

Stimulus materials-Form and size. Two outline forms (circle, square) and three sizes (small, medium, large) were used. The physical dimensions of the stimuli were the same as in Experiment 1. Stimuli were presented in a four-channel ICONIX tachistoscope automated by a solid-state logic system. At a viewing distance of $91 \mathrm{~cm}$, the small, medium, and large figures subtended visual angles of $.4, .8$, and $1.6 \mathrm{deg}$, respectively. In the simultaneouscomparison task, the stimulus pairs were arranged horizontally such that the center of each stimulus appeared $.9 \mathrm{deg}$ from the fixation point. In order to equate the eccentricity of stimulus presentation in the speeded-classification task, the center of each individually presented stimulus appeared either $.9 \mathrm{deg}$ to the right or left of the central fixation point. Response time was measured to the nearest millisecond starting from stimulus onset.

In the speeded-classification task, the single-dimension condition contained three blocks of 36 trials each: one block of all small-, one block of all medium-, and one block of all largesized circles and squares. In each block, half of the stimuli appeared on each side of the fixation point. Therefore, each block of trials in the single-dimension condition contained four different stimuli-2 (forms) by 2 (locations). In order to obtain an equal number of RT measures, the orthogonal condition consisted of three blocks of 36 trials each. Within each block, 18 circles and 18 squares were presented. Half of the six form-size combinations appeared to the right of the fixation point and half appeared to the left. Thus, each block of the orthogonal condition contained 12 different stimuli-2 (forms) by 2 (locations) by 3 (sizes).

Within each biock of trials, each stimulus appeared equally often. In the three single-dimension blocks, each stimulus was followed by itself and by each of the other stimuli an equal number of times. The probability of repeating the same stimulus on Trial $n$ as was presented on Trial $n-1$ was .25 . In the three orthogonal blocks, the probability of repeating the same stimulus from one trial to the next was approximately .08 . The stimuli were presented in a pseudorandom order while adhering to this constraint. Thus, stimulus repetitions were about three times as frequent in the single-dimension blocks of trials.

The simultaneous-comparison task also contained three singledimension blocks of 36 trials each; one block for each level of size. Each block contained four distinct stimulus pairs which were generated by pairing each of the two forms (circle and square) with itself and the other form: (1) circle-circle, (2) square-square, (3) circle-square, and (4) square-circle. Within each block, each of the four pairs of stimuli was repeated nine times, resulting in 18 "same" and 18 "different" trials.

For the orthogonal condition, six different figures were created by combining the two forms with the three sizes. Each of the six figures was then paired with itself and the other five figures to generate a set of 36 distinct pairs of stimuli, consisting of 18 "same" and 18 "different" pairs. Each of the 18 "same" pairs was assigned to one of three categories, according to the relative size difference within a pair: (a) zero level of size disparity (e.g., a pair of medium squares), (b) one level of size disparity (e.g., a medium and a large circle paired), and (c) two levels of size disparity (e.g., a large and a small circle paired). Each of the 18 "differene" pairs was also assigned to one of the three sizedisparity categories. For example, the pair containing a large circle and a small square was assigned to the two-levels-of-size-disparity category. This assignment strategy resulted in an unequal number of same-different pairs in the three size-disparity categories. Specifically, six "same" and six "different" pairs were assigned to the zero size-disparity category, eight "same" and eight "different" pairs were assigned to the one-level-of-size-disparity category, and four "same" and four "different" pairs were assigned to the twolevels-of-size-disparity category. Each of the three orthogonal blocks contained 36 trials. In order to equate the number of stimuhus alternatives with that in the speeded-classification task, only 12 stimulus pairs-two "same" and two "different" pairs from each of the three size-disparity categories-appeared in each orthogonal block of trials. The stimulus pairs were selected such that each of the "same" and "different" pairs within the zero sizedisparity category was included in one of the three orthogonal blocks. However, two of the "same" pairs and two of the "different" pairs from the one-level-of-size-disparity category were not presented in any of the trial blocks, and two of the "same" pairs and two of the "different" pairs from the two-levels-of-sizedisparity category were included in two of the trial blocks. The repeated usage of some, and omission of other, stimulus pairs was determined randomly. 
Within a block of trials, each stimulus pair occurred equally often. In the single-dimension blocks, each pair was followed by itself and by each of the other pairs an equal number of times. The probability of a pair repetition from one trial to the next was approximately .25 . The probability of a pair repetition in each of the orthogonal blocks was approximately .08 . Again, approximately three times as many stimulus repetitions appeared in a single-dimension block as occurred in an orthogonal block.

In summary, the speeded-classification and simultaneouscomparison tasks were equated in terms of: (1) stimulus alternatives ( 4 and 12 different stimuli) in the single-dimension and orthogonal blocks, respectively, (2) stimulus repetitions (three times as many repetitions in the single-dimension as in the orthogonal blocks), and (3) response probabilities.

Stimulus materials-Form and shading. Two values of form (circle, square) and three levels of shading (light, medium, and dark) were used. The physical dimensions of the stimuli were identical to those used in Experiment 1. Each form within a stimulus pair subtended a visual angle of $1.6 \mathrm{deg}$. The individual stimuli in the speeded-classification task and the stimulus pairs in the simultaneous-comparison task were presented in exactly the same manner as described above for the size-irrelevant group.

Procedure. Six subjects were assigned randomly to a sizeirrelevant group, while the remaining six subjects participated in a shading-irrelevant group. Each subject participated in two experimental sessions conducted on successive days. One session was devoted to the speeded-classification task and the other session was devoted to the simultaneous-comparison task. The order of the two tasks was counterbalanced across subjects. Subjects were informed of the nature of the stimulus materials and were shown the stimuli that would appear in the single-dimension and orthogonal blocks of trials. In the speeded-classification task, subjects were instructed to make either a "circle" or a "square" response on each trial. In the simultaneous-comparison task, subjects made either a "same" or "different" response on each trial. Subjects were instructed to respond as quickly and accurately as possible in all conditions of both tasks. They were instructed to ignore any variations in size (or shading) that might occur and to base their responses on only the forms of the stimuli. Subjects responded by pressing one button with the index finger of their preferred hand and another button with the index finger of their nonpreferred hand. ${ }^{3}$ Half of the subjects used their preferred hand to make a "circle" response in the speeded-classification task and a "same" response in the simultaneous-comparison task.

For both tasks, the order of the three single-dimension and three orthogonal blocks was arranged according to a 6 (subjects) by 6 (blocks) Latin square. During each session, each subject received 18 blocks of trials, three repetitions of each of the six blocks. This was obtained by running the subject through the same block sequence three times. The first run through the six blocks served as practice. A 5 -min break was provided after nine blocks of trials. A subject received the same block order in both the speeded-classification and simultaneous-comparison tasks.

The same sequence of events occurred on each trial in both tasks. The subjects focused on a fixation point located in the center of their visual field. Meanwhile, a ready signal was presented in the form of a $500-\mathrm{msec}, 1,000-\mathrm{Hz}$ tone. Half a second later, the fixation point was replaced for $200 \mathrm{msec}$ by a stimulus display. The fixation point then reappeared and a new trial started approximately $1.5 \mathrm{sec}$ after a response was made.

\section{Results and Discussion}

Speeded-classification reaction times. Overall performance was analyzed separately for the size- and shading-irrelevant groups by entering the mean correct $\mathrm{RT}$ s from the three single-dimension conditions and the orthogonal condition into a 2 (responses) by 4 (conditions) by 6 (subjects) repeated-measures analysis of variance. Orthogonal interference caused by

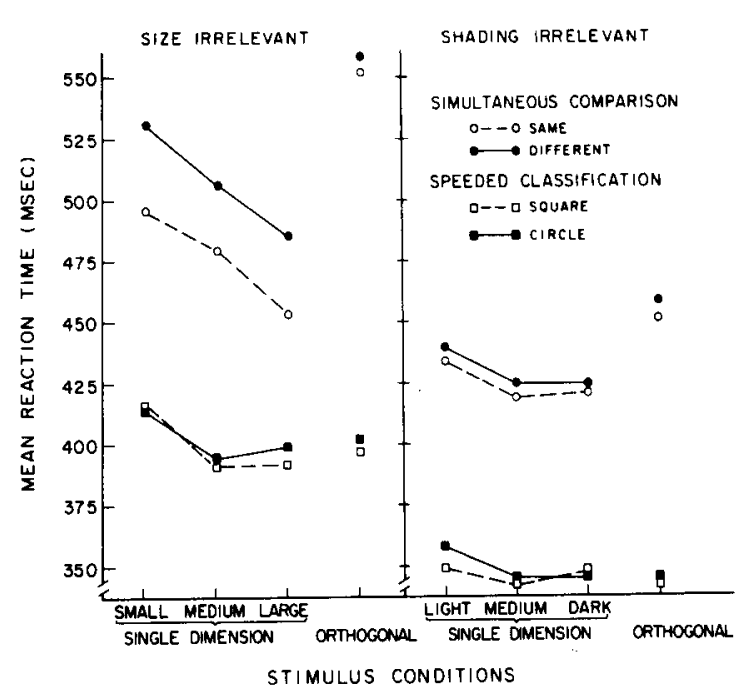

Figure 1. Mean reaction time in the size-irrelevant and shadingirrelevant groups of Experiment 2. Times are shown as a function of the single-dimension and orthogonal conditions of the speededclassification and simultaneous-comparison tasks.

variation on the irrelevant dimension was tested in each group by subtracting RT averaged across the single-dimension conditions from mean RT in the orthogonal condition.

The speeded-classification latencies for the sizeirrelevant group are shown in the lower portion of the left panel of Figure 1. Overall analysis of the response latencies revealed only a significant main effect of conditions $[F(3,15)=5.80, p<.01]$. Response times were longer in the small condition than in either the medium $[\mathrm{t}(5)=4.27, \mathrm{p}<.01]$ or large $[\mathrm{t}(5)=2.86$, $\mathrm{p}<.05$ ] conditions, an effect due possibly to a limitation in visual acuity in the small condition. The minor RT difference between the single-dimension (401 msec) and orthogonal (399 msec) conditions was not significant $[t(5)=.30, p>.20]$. This lack of orthogonal interference confirms the results of Experiment 1 , indicating that subjects are able to attend selectively to form when size varies.

The right panel of Figure 1 depicts the results when shading was the irrelevant dimension in the speededclassification task. In this case, there were no significant differences among conditions. This means, of course, that there was no orthogonal interference. Thus, subjects can attend selectively to form when shading varies.

Repetition effects were also examined in the size- and shading-irrelevant speeded-classification tasks. In both the single-dimension and orthogonal conditions, mean RTs were determined for trials in which: (1) both the stimulus and response from the previous trial were repeated, (2) only the response was repeated, and (3) neither the stimulus nor the response was repeated. The mean RTs were then entered into a 2 (conditions) by 3 (transitions) by 6 (subjects) repeated-measures analysis of variance. Neither the 
main effects nor the interaction between conditions and transitions attained significance in the sizeirrelevant and shading-irrelevant groups $(p>.20)$. The lack of repetition effects fails to support Dixon and Just's (1978) proposal that a subject compares the stimulus presented on Trial $\mathrm{n}$ with his representation of the stimulus presented on Trial $n-1$ in a speeded-classification task.

Simultaneous-comparison reaction times. Overall performance in the size- and shading-irrelevant groups was analyzed by means of separate 2 (responses) by 4 (conditions) by 6 (subjects) repeatedmeasures analyses of variance. Two different measures of interference were obtained in each group: (1) interference due to between-trial variation in which levels of the irrelevant dimension varied from one trial to the next, but were the same for both forms within a stimulus pair (this type of variation occurred in the zero-disparity category), and (2) interference due to within-trial variation in which levels of the irrelevant dimension varied between forms within a stimulus pair (this type of variation occurred in the one- and two-levels-of-disparity categories). Between-trial interference was measured by subtracting RT averaged across the three single-dimension conditions from mean $\mathrm{RT}$ in the zero-disparity category within the orthogonal condition. Between-trial variation was measured separately for the "same" and "different" responses from each subject. Withintrial interference was measured by subtracting mean $\mathrm{RT}$ in the zero-disparity category from RT averaged across the one- and two-levels-of-disparity categories within the orthogonal condition. Separate measures of within-trial interference were also obtained for "same" and "different" responses from each subjext.

The simultaneous-comparison RTs for the sizeirrelevant group are presented in the upper portion of the left panel of Figure 1. The overall analysis showed a significant main effect of conditions $[F(3,15)=40.31$, $p<.001]$ and responses $[F(1,5)=19.51, p<.01]$. "Same" judgments were faster than "different" judgments (by approximately $25 \mathrm{msec}$ ), an effect reported by a number of investigators (e.g., Bamber, 1969; Posner \& Boies, 1971; Tversky, 1969). Both "same" $[\mathrm{t}(5)=5.86, \mathrm{p}<.01]$ and "different" [t(5) $=4.21, p<.01]$ response latencies were longer in the small condition than in the large, single-dimension condition. Again, this difference may be attributed to factors concerning visual acuity. The interaction between conditions and responses was also significant $[\mathrm{F}(3,15)=3.46, \mathrm{p}<.05]$. As shown in Figure 1, "same" RT was approximately $30 \mathrm{msec}$ faster than "different"' RT in each of the single-dimension conditions, whereas only $5 \mathrm{msec}$ separated the two responses in the orthogonal condition. This is analogous to Egeth and Blecker's (1971) finding that the latency of "same" judgments increases more than "different" latencies to pairs of stimuli that are unfamiliar or difficult to compare.

For the size-irrelevant group, the magnitude of between-trial interference (mean RT in the zerodisparity category of the orthogonal condition minus RT averaged across the single-dimension conditions) was $25 \mathrm{msec}$ for "same" judgments $[\mathrm{t}(5)=1.95$, $\mathrm{p}>.10$ ] and $48 \mathrm{msec}$ for "different" judgments $[t(5)=9.84, p<.001]$. The faster RTs obtained in the single-dimension conditions may be attributed in part to the fact that subjects knew that only levels on the relevant dimension could vary in those conditions. However, when levels on both the relevant and irrelevant dimensions varied in the orthogonal condition, subjects might have employed some type of inhibitory or checking operation to assure that they were responding to only the relevant dimension (cf. Eriksen \& Eriksen, 1974). This operation, which appears to be more prominent in "different" judgments, results in longer RT.

The magnitude of within-trial interference (RT averaged across the one- and two-levels-of-disparity categories minus mean $\mathrm{RT}$ in the zero-disparity category of the orthogonal condition) in the size-irrelevant group was $69 \mathrm{msec}$ for "same" judgments [t(5)= 7.37, $p<.001]$ and $1 \mathrm{msec}$ for "different" judgments $[\mathrm{t}(5)=.10, \mathrm{p}>.40]$. In short, size disparity within trials affected only "same" judgments, whereas size variations between trials primarily affected "different" judgments. Overall, these results point to the fact that although interference was not obtained in the speeded-classification task, subjects were unable to filter out variations in size in the simultaneouscomparisor task without concomitant increases in response latency.

The simultaneous-comparison RTs for the shadingirrelevant group are presented in the upper part of the right panel of Figure 1. The overall analysis showed only a significant main effect of conditions $[F(3,15)=6.61, p<.005]$. Unlike the case in the sizeirrelevant group, the main effect of responses and the interaction between conditions and responses were not significant. We have no explanation to offer for these differences between the size-irrelevant and shading-irrelevant groups.

Reaction time for the shading-irrelevant group was longer in the orthogonal condition than in the singledimension conditions for both "same" and "different" responses. The magnitude of between-trial interference was only $5 \mathrm{msec}$ for "same" judgments $[\mathrm{t}(5)=.58, \mathrm{p}>.20]$ and $31 \mathrm{msec}$ for "different" judgments $[\mathrm{t}(5)=4.08, \mathrm{p}<.001]$. Measures of withintrial interference were much greater for "same" (30 msec) judgments $[\mathrm{t}(5)=4.35, \mathrm{p}<.001]$ than for "different" $(-9 \mathrm{msec})$ judgments $[\mathrm{t}(5)=1.59, \mathrm{p}>.10]$. In other words, between-trial variation affected only "different" judgments, whereas within-trial varia- 
tion affected only "same" judgments. This pattern of results was also obtained for the size-irrelevant group and is consistent with the notion that separate processes may be responsible for the two types of perceptual judgments (cf. Bamber, 1969; Tversky, 1969).

Errors. Percentages of erroneous responses across conditions and responses in the speeded-classification and simultaneous-comparison tasks appear in Table 2. The error rate was low in all conditions, ranging from less than $1 \%$ to $4 \%$ for individual subjects. Most importantly, there were no significant differences in error rates between responses or conditions in either the speeded-classification or simultaneouscomparison tasks ( $\mathrm{p}>.20$ ).

Normalization. Analysis of the response latencies in the orthogonal condition revealed a significant interaction between levels of disparity and responses in both the size-irrelevant $[\mathrm{F}(2,10)=14.50, \mathrm{p}<.005]$ and shading-irrelevant $[\mathrm{F}(2,10)=12.67, \mathrm{p}<.005]$ groups. This interaction is consistent with the fact that within-trial interference was obtained for only "same" judgments in the simultaneous-comparison tasks.

What is of greatest interest is that mean "same" $\mathrm{RT}$ increased with corresponding increments in the amount of disparity on the irrelevant dimension in both the size-irrelevant $[F(2,10)=26.23, p<.001]$ and shading-irrelevant $[\mathrm{F}(2,10)=21.15, \mathrm{p}<.001]$ groups. This can be seen in Figure 2. Since level of disparity is an ordinal dimension, normalization was expected to produce a monotonic increase in RT. The Page test of monotonicity (Marascuilo \& McSweeney, 1977, pp. 374-375) revealed a significant monotonic increase in RT with corresponding increases in disparity on the irrelevant dimensions of size $(p<.01)$ and shading $(p<.01)$. Thus, it is clear that the time required to judge whether two forms are the same increases monotonically with increases in the amount of disparity on the irrelevant dimension. Relative size differences produced much greater increments in

Table 2

Mean Percentage of Errors for Each Condition in Experiment 2

\begin{tabular}{|c|c|c|c|c|}
\hline \multirow[b]{2}{*}{ Condition } & \multicolumn{2}{|c|}{$\begin{array}{l}\text { Speeded Classi- } \\
\text { fication }\end{array}$} & \multicolumn{2}{|c|}{$\begin{array}{l}\text { Simultaneous } \\
\text { Comparison }\end{array}$} \\
\hline & Circle & Square & Same & Different \\
\hline & \multicolumn{4}{|c|}{ Size-Irrelevant Dimension } \\
\hline $\begin{array}{l}\text { Small } \\
\text { Medium } \\
\text { Large }\end{array}$ & $\begin{array}{r}.93 \\
3.24 \\
.46\end{array}$ & $\begin{array}{r}1.85 \\
1.39 \\
.93\end{array}$ & $\begin{array}{r}2.31 \\
.93 \\
.93\end{array}$ & $\begin{array}{l}2.31 \\
1.85 \\
2.78\end{array}$ \\
\hline \multirow[t]{2}{*}{ Orthogonal } & 1.54 & 1.54 & 3.24 & 1.23 \\
\hline & \multicolumn{4}{|c|}{ Shading-Irrelevant Dimension } \\
\hline $\begin{array}{l}\text { Light } \\
\text { Medium } \\
\text { Dark }\end{array}$ & $\begin{array}{l}1.85 \\
3.70 \\
3.24\end{array}$ & $\begin{array}{l}2.31 \\
2.78 \\
2.78\end{array}$ & $\begin{array}{r}3.70 \\
.93 \\
3.70\end{array}$ & $\begin{array}{l}2.31 \\
1.85 \\
1.85\end{array}$ \\
\hline Orthogonal & 3.40 & 1.54 & 3.86 & 2.01 \\
\hline
\end{tabular}

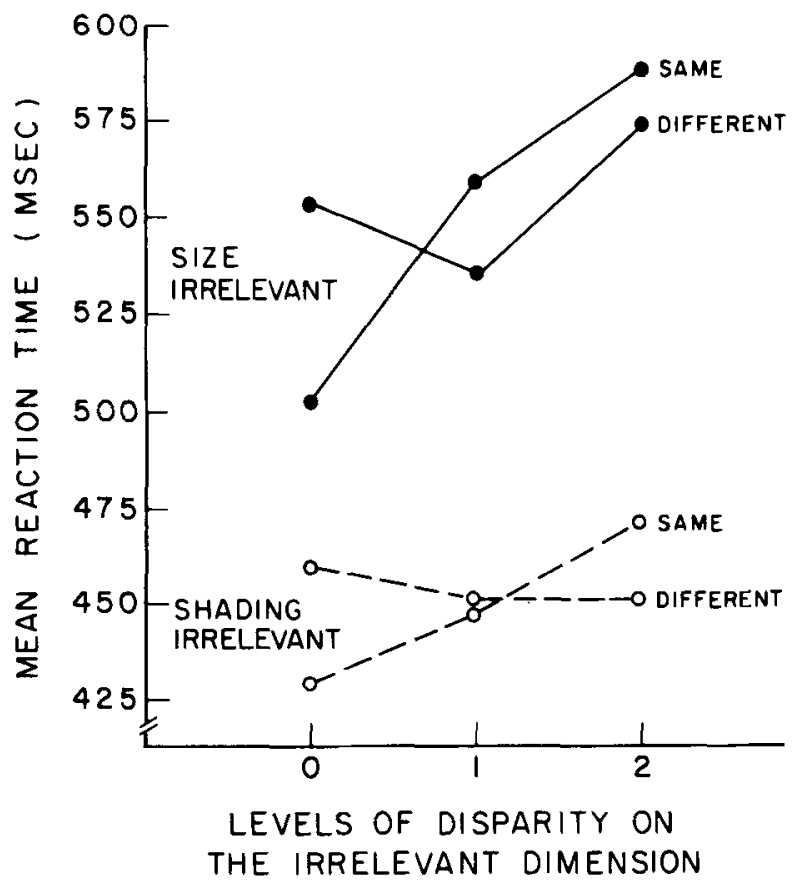

Figure 2. Mean "same" and "different" reaction times as a function of the levels of disparity on the irrelevant dimensions in Experiment 2.

"same" RT than did relative differences in shading. This suggests that the psychological differences among the levels of size that we chose were greater than the differences among the three levels of shading available to us. It would be inappropriate to conclude, however, that relative size differences necessarily result in larger amounts of graded interference. It is likely that shading could create equivalent, if not larger, amounts of graded interference, with more extreme physical differences.

"Different" judgments, as shown in Figure 2, did not vary systematically as a function of the amount of irrelevant disparity. Other investigators have also failed to obtain an effect of irrelevant disparity on "different" responses in the study of mental size scaling (e.g., Besner \& Coltheart, 1975, 1976; Corcoran \& Besner, 1975; Sekuler \& Nash, 1972) and mental rotation (e.g., Shepard \& Metzler, 1971). Dixon and Just (1978) have also shown normalization of qualitative dimensions such as color to occur on "same" but not on "different" trials.

\section{EXPERIMENT 3}

The results of Experiment 2 indicate that the ability to attend selectively to a relevant dimension is task dependent. Both speeded classification and simultaneous comparison required subjects to develop an internal representation or image of the figure(s) presented on each trial. In the speeded-classification task, the image is then compared with representa- 
tions (or names) in memory that are mapped onto particular responses. In view of the lack of orthogonal interference, the memorial representations appear to be in a size- and shading-free format. Furthermore, the lack of repetition effects in the speededclassification task failed to support the proposal that the image of a figure on each trial was compared against the representation of the stimulus on the previous trial (cf. Felfoldy, 1974). One might argue that interference resulting from repetition effects would have been obtained if an RSI comparable to Felfoldy's had been used. Recall, however, that Experiment 1 employed a card-sorting task (with the cards held faceup). In this situation the RSI is very short, yet no interference was obtained.

The simultaneous-comparison task also requires an initial stage during which each figure is encoded. Measures of within- and between-trial interference indicate that subjects do not compare the representation of each figure against size- and shading-free representations in memory, but they appear to directly compare the two representations in order to determine if they are the same or different with respect to form. Apparently, the overall disparity between the two stimuli in the orthogonal condition is so compelling that it takes a subject some time to overcome the difference on the irrelevant dimension before a "same" judgment based on form alone can be completed. In fact, the time required for "same" judgments was found to increase monotonically as a function of the amount of disparity on the irrelevant dimension.

In summary, the primary difference between speeded classification and simultaneous comparison appears to be that subjects do not make a direct comparison between stimuli on successive trials in the speededclassification task, even when the RSI is extremely short (see the card-sorting task in Experiment 1). Based on the results of Experiment 2, it might appear that a comparison between stimuli that results in interference and normalization is dependent on the simultaneous presentation of the to-be-compared stimuli. However, other investigators have also reported the normalization of size when it is the irrelevant dimension in a successive-comparison paradigm (e.g., Besner \& Coltheart, 1976; Larsen \& Bundesen, 1978). In that task, the visual representation of the first stimulus is retained until the second stimulus is presented, at which time the image of the first is transformed to the size format of the second stimulus.

Experiment 3 was conducted in order to determine whether subjects would normalize size disparities when stimuli were presented successively. In both of the successive-comparison studies mentioned above, the stimuli that were used had no apparent name association, making it difficult for subjects to encode the first stimulus in a size-free format. However, with easily identified and nameable stimuli such as circles and squares, subjects could compare stimuli based on a representation that is insensitive to size differences (e.g., a name code). Under such circumstances, normalization would not be expected. If, however, subjects were to base their "same" judgments on the same type of visual representation used during simultaneous comparison, then normalization would again be expected.

\section{Method}

Subjects. Six undergraduates at Johns Hopkins University served as paid subjects. Each had normal or corrected-to-normal vision. None of the subjects had participated in Experiment 1 or Experiment 2.

Stimuli. Two outline forms (circle, square) and three sizes (small, medium, large) were used. The physical dimensions of the stimuli were the same as those used in Experiments 1 and 2. The center of each individually presented stimulus coincided with a centrally located fixation point. Six figures were created by combining the two forms with the three sizes. Each of these six figures was then paired with itself and the other five figures in order to generate a set of 36 distinct pairs of stimuli-18 "same" and 18 "different" pairs. As in Experiment 2, each of the 18 "same" and 18 "different" pairs was assigned to one of the three sizedisparity categories - (a) zero level of size disparity, (b) one level of size disparity, and (c) two levels of size disparity. Each block of trials contained a randomized order of 36 stimulus pairs-six "same" and six "different" pairs from each of the three sizedisparity categories. Each of the six "same" and six "different" pairs within the zero-size-disparity category appeared within each block of trials. However, two "same" and two "different" pairs from the one-level-of-size-disparity category were not included in each block of trials and two "same" and two "different" pairs from the two-levels-of-size-disparity category were repeated once within each block of trials. The repetition of stimulus pairs and the exclusion of pairs from a block of trials were determined randomly.

Procedure. Each form within a pair was presented individually. On each trial, while the subject focused on the fixation point, a ready signal was presented in the form of a $500-\mathrm{msec}, 1,000-\mathrm{Hz}$ tone. Half a second later, the fixation point was replaced by the first stimulus for $200 \mathrm{msec}$. After a blank interstimulus interval (ISI) of $0,100,300,500,1,000$, or $2,000 \mathrm{msec}$, the second stimulus was presented. As in previous investigations using the successivecomparison task (e.g., Besner \& Coltheart, 1976; Larsen \& Bundesen, 1978), the second stimulus remained on until the subject responded. Response time was measured to the nearest millisecond starting with the onset of the second stimulus. Subjects were instructed to determine as quickly and accurately as possible whether the two stimuli had the "same" or "different" forms and to ignore any variations in size that might occur. They responded by pressing one button with the index finger of their preferred hand and another button with the index finger of their nonpreferred hand. Half of the subjects used their preferred hand to respond "same."

The order of the six ISIs was arranged according to a 6 (subjects) by 6 (ISIs) Latin square. During the experimental session, a subject received 18 blocks of trials-three blocks at each of the six ISIs. All three blocks of trials were provided at one ISI before switching to a new ISI. Ten practice trials were provided at each of the ISIs.

\section{Results and Discussion}

Shown in Table 3 are the mean reaction times and error percentages obtained in Experiment 3. The mean RTs from each subject were entered into a 3 (levels of size disparity) by 2 (responses) by 6 (ISIs) 
Table 3

Mean Reaction Time (RT) in Milliseconds and Percentage of Errors (E) for Each Level of Size Disparity, Response Type, and Interstimulus Interval (ISI) in Experiment 3

\begin{tabular}{|c|c|c|c|c|c|c|c|c|c|c|c|c|c|c|c|c|}
\hline \multirow[b]{3}{*}{ ISI } & \multicolumn{8}{|c|}{ Same } & \multicolumn{8}{|c|}{ Different } \\
\hline & \multicolumn{2}{|c|}{0} & \multicolumn{2}{|c|}{1} & \multicolumn{2}{|c|}{2} & \multicolumn{2}{|c|}{ Mean } & \multicolumn{2}{|c|}{0} & \multicolumn{2}{|c|}{1} & \multicolumn{2}{|c|}{2} & \multicolumn{2}{|c|}{ Mean } \\
\hline & RT & E & RT & E & RT & E & RT & E & RT & E & RT & E & RT & E & RT & $\mathrm{E}$ \\
\hline 0 & 447 & 1.9 & 473 & 3.7 & 495 & 1.0 & 472 & 2.2 & 489 & 1.9 & 492 & 2.8 & 503 & 1.9 & 495 & 2.2 \\
\hline 100 & 416 & 1.0 & 446 & 1.0 & 476 & 2.8 & 446 & 1.6 & 466 & .0 & 472 & 1.9 & 48 & 1.0 & 475 & 1.0 \\
\hline 300 & 424 & .0 & 465 & 1.0 & 469 & 1.9 & 453 & 1.0 & 483 & 1.9 & 477 & 1.0 & 482 & 3.7 & 481 & 2.2 \\
\hline 500 & 431 & 1.9 & 446 & .0 & 467 & .0 & 448 & .6 & 498 & 1.9 & 483 & 1.9 & 491 & 2.8 & 491 & 2.2 \\
\hline 1,000 & 480 & 1.9 & 489 & 1.9 & 496 & 1.0 & 488 & 1.6 & 532 & .0 & 515 & 1.9 & 542 & 1.0 & 530 & 1.0 \\
\hline 2,000 & 481 & 1.0 & 510 & 1.9 & 495 & 4.6 & 495 & 2.5 & 541 & 3.7 & 525 & 4.6 & 553 & 1.9 & 540 & 3.4 \\
\hline Mean & 447 & 1.3 & 472 & 1.6 & 483 & 1.9 & & & 501 & 1.6 & 494 & 2.4 & 510 & 2.1 & & \\
\hline
\end{tabular}

by 6 (subjects) repeated-measures analysis of variance. Errors were also analyzed in the same manner. No significant differences were obtained in the error data $(\mathrm{p}>.20)$. In the RT data, the main effect of size disparity was significant $[F(2,10)=21.26, \mathrm{p}<.001]$, revealing a general trend for $\mathrm{RT}$ to increase with corresponding increases in size disparity. The interaction between size disparity and responses was also significant $[F(2,10)=8.11, \mathrm{p}<.01]$. Inspection of Table 3 reveals that the monotonic increase in RT was more pronounced for "same" than for "different" judgments. Overall, "same" RT (467 msec) was significantly faster than "different" RT (502 msec) $[F(1,5)=10.54, p<.025]$. No other main effects or interactions attained significance, except the marginally significant interaction between size disparity and ISI $[F(10,50)=1.84, .05<p<.10]$, which suggests that the effects of size disparity were greater at the shorter ISIs. ${ }^{4}$

Figure 3 depicts the amount of within-trial interference attributable to size disparity as a function of ISI and response. As in Experiment 2, this measure of interference was obtained by subtracting mean RT in the zero-disparity category from RT averaged across the one- and two-levels-of-disparity categories. The interference functions for the "same" and "dif-

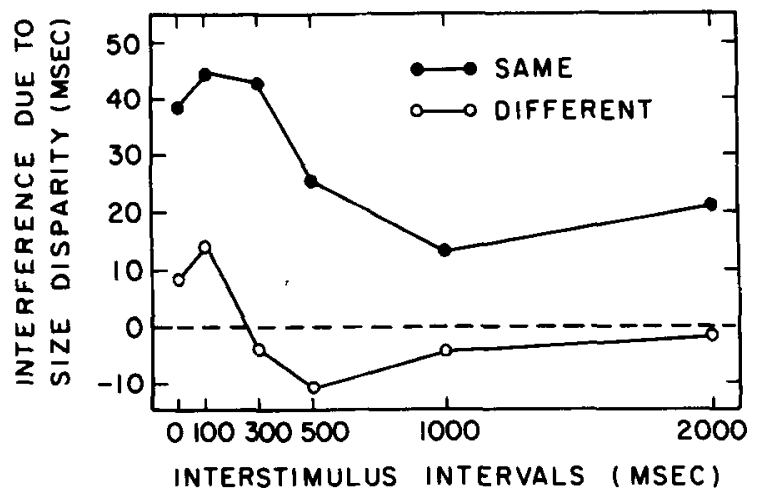

Figure 3. Within-trial interference as a function of interstimulus intervals (ISIs) and responses in Experiment 3. ferent" judgments shown in Figure 3 are quite similar to one another in that both show a greater amount of interference at the shorter ISIs. Post hoc analyses revealed a significant amount of interference at each of the 0-, 100-, 300-, and 500-msec ISIs for "same" judgments $(p<.01)$. "Different" judgments, however, were not affected significantly at any of the ISIs $(p>.10)$. These results are consistent with the findings obtainer in the simultaneous-comparison task of Experiment 2 in that only "same" judgments were affected by size disparity within stimulus pairs.

Normalization of size disparity occurred on "same" trials at the 0- and 100-msec ISIs. As shown in Table 3, mean "same" RT increased with corresponding increments in the amount of size disparity at both the 0 -msec ISI $[F(2,10)=8.00, p<.01]$ and the 100 -msec ISI $[F(2,10)=56.24, p<.001]$. The Page test of monotonicity also revealed a significant monotonic increase in RT with corresponding increases in size disparity at both ISIs $(p<.001)$. These results demonstrate that simultaneous presentation is not a necessary condition for the normalization of irrelevant dimensional disparity.

Although interference was obtained at the 300- and 500 -msec ISIs, subjects did not produce a reliable monotonic increase in "same" RT; only three of the six subjects produced a monotonic increase in $\mathrm{RT}$ at the $300-\mathrm{msec}$ ISI and only two of six produced a monotonic increase in RT at the $500-\mathrm{msec}$ ISI. At both the 300- and 500-msec ISIs, RT was significantly faster in the zero-level-of-disparity category $(p<.025)$, but did not differ between the one- and two-levels-of-disparity categories $(p>.20)$.

The results from Experiment 3 may be interpreted by first assuming that when a common geometric shape is presented visually, both a physical code and size-free nominal code are developed internally (cf. Posner, 1978). Although the "visual" and "name" codes of a stimulus may be accessed in parallel, our data suggest that the hasis for matching nameable geometric shapes changes over time. Interference due to size disparity obtained at ISIs from 0 to $500 \mathrm{msec}$ 
suggests that subjects match shapes based on some type of visual repiesentation of the first stimulus. The monotonic increase in "same" RT at the 0- and 100 -msec ISIs is consistent with an extension of the Dixon-Just normalization model, in which it is assumed that subjects internally transform the size format of the first stimulus to match that of the second stimulus before a "same" judgment is made. "Same" judgments at the 300- and 500-msec ISIs, however, appear to be based on both a visual and size-free name code. The faster responses in the zerodisparity category suggest that "same" judgments are based on a low-level perceptual match when the visual representation of the first stimulus matches the format of the second stimulus. However, when irrelevant disparity is present and the visual representation of the first stimulus does not match the format of the second stimulus, subjects appear to base their "same" judgments on a higher, size-free name code. Reaction times in the one- and twolevels-of-disparity categories are approximately the same, suggesting that the time required to compare the two stimuli based on a name code for each is independent of the amount of disparity between them. Finally, the lack of interference in the "same" judgments at the 1- and 2-sec ISIs suggests that subjects may have compared stimuli based on a sizefree, name code.

\section{GENERAL DISCUSSION}

\section{Speeded Classification and Comparison}

Experiments 2 and 3 demonstrated that when subjects compared multidimensional stimuli that were presented either simultaneously or with a short delay between them, time was required to filter out disparity on the irrelevant dimension. Specifically, "same" RT increased monotonically with corresponding increases in irrelevant disparity. When stimuli were presented in the speeded-classification task, no evidence of a comparison process was obtained. Furthermore, irrelevant dimensional disparity was ignored without increasing the time required to make form classifications.

The differences between speeded-classification and comparison tasks noted above suggest that the distinction between integral and separable stimulus dimensions may depend on the specific task employed. In most cases, dimensional combinations are deemed to be integral or separable based on the results of the correlated and orthogonal conditions of a speededclassification task. Consequently, most processing models are limited to the identification and classification of individually presented stimuli (see, e.g., Lockhead, 1979). We did not perform all the tests of integrality and separability, since we were primarily interested in the processes affecting selective attention in the two tasks. Nevertheless, the fact that orthogonal interference was not obtained in the speeded-classification task suggests that the dimensions of form and size as well as form and shading are separable. Yet, these same dimensions generated interference in similar conditions of the comparison tasks in which subjects made speeded judgments of the perceived relations between stimuli. In short, the ability to attend selectively to a stimulus dimension is not simply a function of the particular stimulus dimensions employed, but is also a function of the particular task and processing strategies required of the sub; sct (see Dykes, 1979, for a similar argument).

The results of Experiment 2 fail to support Dixon and Just's (1978) proposal that a common process underlies interference in the speeded-classification and comparison tasks. If interference in both tasks were mediated by the same process, then the same stimulus dimensions presented under comparable conditions would produce similar results in the two tasks. Our results indicate that even though no evidence of orthogonal interference or comparisons between successive stimuli was obtained in the speededclassification task, the same stimulus dimensions resulted in sizable interference effects in both the simultaneous-comparison and successive-comparison tasks. Furthermore, our data indicate that normalization of irrelevant dimensional disparity occurs even when: (1) the levels of the irrelevant dimension may be accessed easily through a set of encoding features such as the names of common geometric figures and (2) the stimuli are presented in a successivecol.uparison paradigm.

Normalization. According to Dixon and Just (1978), the monotonic increase in "same" RT with corresponding increases in irrelevant disparity indicates that "subjects mentally equate two stimuli on the irrelevant dimension before deciding that they are identical along the relevant dimension"' (p. 37). Dixon and Just have noted that alternative accounts of interference effects are available, but none of them appear to be as comprehensive in accounting for the results from the comparison studies. Some possible alternative explanations are discussed in the following paragraphs.

(1) A categorization model predicts that when no irrelevant disparity is present, "same" responses are based on a fast template or physical match. However, when irrelevant disparity is present, time is required to reach a higher categorical or name code upon which a response is based. Since the time required for categorization is not affected by the amount of disparity, this model predicts no difference between responses when small and large amounts of disparity are present. The categorization model accounts quite nicely for the effects of size disparity on "same" RT at the 300- and 500-msec ISIs in the successive-comparison task of Experiment 3. However, "same" RT increased significantly with each 
additional level of disparity in the simultaneous- and successive-comparison tasks at the $0-$ and $100-\mathrm{msec}$ ISIs.

(2) Hawkins and Shigley (1972) have also suggested that when no irrelevant disparity is present, a low-level perceptual match can be made, since the distinction between relevant and irrelevant dimensions is of no consequence. However, when two stimuli do not match, it becomes necessary to consult memory to determine which dimension is relevant and which is irrelevant in order to make a correct response. Since the interrogation of memory is dependent on the detection of a mismatch, the sooner a mismatch is detected, the faster the correct response can be determined. Therefore, this model predicts that greater amounts of disparity should produce quicker detections of mismatches and hence faster "same" responses, a prediction opposite to what was found in this study.

(3) The response-competition model also provides another popular explanation of interference caused by irrelevant variation (Dykes, 1979; Egeth, 1966). According to Dykes, relevant and irrelevant dimensions are processed by "separate dimensional analyzers" and interference results from the misallocation of attention to the irrelevant dimensional analyzer whenever it produces an output that is incompatible with the output of the relevant dimensional analyzer. Thus, a difference on the irrelevant dimension competes with the required "same" response such that the greater the amount of disparity, the greater the competition. This model also predicts that "different" responses should be graded. That is, increases in irrelevant disparity should result in faster "different" responses. Although Dykes has reported interference due to incompatible outputs for both "same" and "different" responses when accuracy is measured, we and other investigators have failed to find any systematic relationship between "different" responses and levels of disparity when $\mathrm{RT}$ is measured (e.g., Dixon \& Just, 1978).

The normalization model appears to provide a more accurate account of the graded interference effects obtained in our comparison tasks than the three models described above. However, our results also indicate that the normalization of irrelevant disparity is not a stage of processing that uniformly precedes the comparison of stimuli along the relevant dimension. If this were the case, then "different" RT would have also increased monotonically with relative differences in size or shading. ${ }^{5}$

The selective effect of irrelevant disparity on "same" judgments is consistent with a dual-process model in which it is assumed that "same" and "different" judgments are mediated by independent processes. Specifically, several investigators have proposed that "same" judgments are mediated by a holistic process that is more sensitive to the configuration of a stim- ulus pair, whereas "different" judgments are determined by a more analytic process that compares stimuli feature by feature (e.g., Bamber, 1969; Egeth \& Blecker, 1971; Krueger, 1973; Nickerson, 1969, 1978).

In this vein, "different" judgments would not be affected by irrelevant disparity if they were based on an ordered comparison process (Besner \& Coltheart, 1976). According to this position, either the relevant dimension is examined prior to the irrelevant dimension (this is similar to Egeth's, 1966, serial, selfterminating model) or both dimensions are processed in parallel but more capacity is allocated to the analysis of the relevant dimension such that it finishes first. In Experiment 2, "different" RT was longer in the zero-disparity category of the orthogonal condition than in the single-dimension conditions. This may have resulted from the extra time required to determine which was the relevant dimension or from the sharing of processing capacity across two stimulus dimensions in the orthogonal condition. The impor tant point is that RT for "different" judgments was independent of the amount of irrelevant disparity within a stimulus pair.

"Same" judgments, however, were affected by irrelevant disparity within stimulus pairs, suggesting that the "same" analyzing mechanism is incapable of filtering out irrelevant differences between stimuli. Insıead of a time-consuming normalization process that precedes "same" judgments, Besner and Coltheart (1976) have suggested that the monotonic increase in "same" RT reflects an increase in the time required to determine the correct response when a difference along the irrelevant dimension is present. According to their criterion-shift model, the "same" analyzing mechanism operates on a hierarchy of perceptual tests. When no irrelevant disparity is present, "same" responses are assumed to be based on a fast template or holistic match at a low level in the perceptual system (see also Bamber, 1969; Hawkins $\&$ Shigley, 1972). If a match is not obtained at this level, then the stimuli are compared along the relevant dimension. In addition, the early detection of a difference along the irrelevant dimension is assumed to raise the criterion for deciding that the two stimuli are the same such that more evidence concerning the relevant dimension is required before a "same" response can be evoked. The monotonic increase in "same" RT can be explained based on the assumption that greater irrelevant differences result in greater criterion shifts.

Several points about the criterion-shift model deserve to be mentioned. First, it should be noted that this model is in better accord with the data than is the response-competition model described earlier, since the latter model cannot account for the fact that irrelevant disparity affects only "same" judgments. Second, the criterion-shift model keeps us 
from having to postulate a stage of processing such as normalization that inexplicably precedes only "same" judgments. Third, the criterion-shift model has a great deal of intuitive appeal, since it is based on the idea that subjects are reluctant to judge two stimuli as being the "same" when a difference exists between them on the irrelevant dimension. The point is that sameness typically implies complete identity. A subject's reluctance to respond "same" to two stimuli differing on the irrelevant dimension may be manifested in the raising of the decision criterion for sameness. Finally, the model can readily account for the data from both the simultaneous- and successivecomparison tasks. The point that needs explaining is why the effect of irrelevant disparity dissipates with time (i.e., with increasing ISI). We suggest that this is a natural consequence of the relatively faster loss of the visual, as opposed to the size-free, name code.

Unfortunately, at the present time it is unknown whether the monotonic increase in "same" RT is the result of an analogue transformation of irrelevant disparity (i.e., normalization) or a shift in decision criteria associated with the sameness mechanism. The distinction between these two models awaits further investigation.

\section{REFERENCE NOTE}

1. Egeth, H. E., \& Gilmore, G. C. Perceptibility of the letters in words and nonwords with complete control of redundancy. Paper presented at the meeting of the Psychonomic Society, St. Louis, Missouri, November 1973.

\section{REFERENCES}

BAmber, D. Reaction times and error rates for "same" - "different" judgments of multidimensional stimuli. Perception \& Psy. chophysics, 1969, 6, 169-174.

Besner, D., \& Coltheart, M. Same-different judgments with words and nonwords: The differential effects of relative size. Memory \& Cognition, 1975, 3, 673-677.

Besner, D., \& Coltheart, M. Mental size scaling examined Memory \& Cognition, 1976, 4, 525-531.

Bundesen, C., \& Larsen, A. Visual transformation of size. Journal of Experimental Psychology: Human Perception and Performance, 1975, 1, 214-220.

Corcoran, D. W. J., \& Besner, D. Application of the Posner technique to the study of size and brightness irrelevancies in letter pairs. In P. M. A. Rabbitt \& S. Dornic (Eds.), Attention and performance $V$. London: Academic Press, 1975.

Dixon, P., \& Just, M. A. Normalization of irrelevant dimensions in stimulus comparisons. Journal of Experimental Psychology: Human Perception and Performance, 1978, 4, 36-46.

DYкES, J. R. A demonstration of selection of analyzers for integral dimensions. Journal of Experimental Psychology: Human Perception and Performance, 1979, 5, 734-745.

Dykes, J. R., \& COOPE R, R. G. An investigation of the perceptual basis of redundancy gain and orthogonal interference for integral dimensions. Perception \& Psychophysics, 1978, 23, 36-42.

Egeth, H. E. Parallel versus serial processes in multidimensional discrimination. Perception \& Psychophysics, 1966, 1, 245-252.

Egeth, H. E., \& Blecker, D. Differential effects of familiarity on judgments of sameness and difference. Perception \& Psychophysics, 1971, 9. 321-326.
Eriksen, B. A., \& Eriksen, C. W. Effects of noise letters upon the identification of a target letter in a nonsearch task. Perception \& Psychophysics, 1974, 16, 143-149.

Felfoldy, G. L. Repetition effects in choice reaction time to multidimensional stimuli. Perception \& Psychophysics, 1974, $15,453-459$

GARNE H, W. R. Attention: The processing of multiple sources of information. In E. C. Carterette \& M. P. Friedman (Eds.), Handbook of perception (Vol. 2). New York: Academic Press, 1974, (a)

GARNER, W. R. The processing of information and structure. Potomac, Md: Erlbaum, 1974. (b)

GARNER, W. R. Interaction of stimulus dimensions in concept and choice processes. Cognitive Psychology, 1976, 8, 98-123.

GarnER, W. R. Selective attention to attributes and to stimuli. Journal of Experimental Psychology: General, 1978, 107, 287-308.

Garner, W. R., \& Felfoldy, G. L. Integrality of stimulus dimensions in various types of information processing. Cognitive Psychology, 1970, 1, 225-241.

Gottwald, R. L., \& Garner, W. R. Effects of focusing strategy on speeded classification with grouping, filtering, and condensation tasks. Perception \& Psychophysics, 1972, 11, 179-182.

Gottwald, R. L., \& Garner, W. R. Filtering and condensation tasks with integral and separable dimensions. Perception \& Psychophysics, 1975, 18, 26-28.

Hawkins, H. L., McDonald, G. J., \& Cox. A. K. Effects of irrelevant information in speeded discrimination. Journal of Experimental Psychology, 1973, 98, 435-437.

Hawkins, H. L., \& Shigley, R. H. Irrelevant information and processing mode in speeded discrimination. Journal of Experimental Psychology, 1972, 96, 389-395.

Keuss, P. J. G. Processing of geometrical dimensions in a binary classification task: Evidence for a dual process model. Perception \& Psychophysics, 1977, 21, 371-376.

KrUEgER, L. E. Effect of irrelevant surrounding material on speed of same-different judgment of two adjacent letters. Journal of Experimental Psychology, 1973, 98, 252-259.

LARSEn, A., \& Bundesen, C. Size scaling in visual pattern recognition. Journal of Experimental Psychology: Human Perception and Performance, 1978, 4, 1-20.

LOCKHEAD, G. R. Holistic versus analytic process models: A reply. Journal of Experimental Psychology: Human Perception and Performance, 1979, 5, 746-755.

Marascullo, L. A., \& McSweeney, M. Nonparametric and distribution-free methods for the social sciences. Belmont, Calif: Wadsworth, 1977.

Massaro, D. W. Perception of letters, words, and nonwords. Journal of Experimental Psychology, 1973, 100, 349-353.

Nickerson, R. S. "Same"."different" response times: A model and a preliminary test. In W. G. Koster (Ed.), Attention and performance II. Amsterdam: North-Holland, 1969.

Nickfrson, R. S. On the time it takes to tell things apart. In J. Requin (Ed.), Attention and performance VII. Hillsdale, N.J: Erlbaum, 1978.

Pomerantz, J. R., \& Garner, W. R. Stimulus configuration in selective attention tasks. Perception \& Psychophysics, 1973, 14, 565-569.

PoSNER, M. I. Information reduction in the analysis of sequential tasks. Psychological Review, 1964, 71, 491-504.

Posner, M. I. Chronometric explorations of mind. Hillsdale, N.J: Erlbaum, 1978.

Posner, M. I., \& Bores, S. J. Components of attention. Psychological Review, 1971, 78, 391.408.

Posner, M. I., \& Mitchell, R. F. Chronometric analysis of classification. Psychological Review, 1967, 74, 392-409.

Sekuler, R., \& Nash, D. Speed of size scaling in human vision. Psychonomic Science, 1972, 27, 93-94.

Shepard, R., \& Metzler, J. Mental rotation of three-dimensional objects. Science, 1971, 171, 701-703. 
TAYLOR, R. L. Comparison of short-term memory and visual sensory analysis as sources of information. Journal of Experimental Psychology, 1969, 81, 515-522.

TVersky, B. Pictorial and verbal encoding in a short-term memory task. Perception \& Psychophysics, 1969, 6, 225-233.

\section{NOTES}

1. It is interesting to note that Massaro (1973, Experiment 2) used a similar set of stimuli in a forced-choice paradigm with single-stimulus presentation and found performance to be equivalent in the word and nonword contexts. This suggests that Massaro's subjects were able to attend selectively to the middle letter position.

2. Dixon and Just (1978) use the term normalization to refer to the process by which subjects internally equate one stimulus with respect to another when a difference exists between them on the irrelevant dimension. According to their explanation, normalization entails an analogue transformation of irrelevant disparity prior to a comparison between identical stimuli on the relevant dimension. This leads to the prediction that "same" RT should increase monotonically as a functicn of disparity on the irrelevant dimension. Throughout this paper, we will use the term normalization only as a convenient way to refer to the monotonic increase in "same" RT without any commitment to Dixon and Just's process model.
3. Response buttons were arranged horizontally, with one in front of the other, in order to eliminate any left-right stimulusresponse compatibility effects in the speeded-classification task.

4. Overall, the response times were faster for the successive comparisons in Experiment 3 than for the simultaneous comparisons in Experiment 2. This may be due to the fact that in the successive-comparison task, one stimulus was already encoded prior to the presentation of the second stimulus and the measurement of RT. However, in the simultaneous-comparison task, RT included the time required to encode two stimuli at once. Other investigators have also reported a successive-comparison RT advantage (e.g., Taylor, 1969).

5. It has occurred to us that the lack of relationship between "different" responses and levels of disparity may represent the net effect of two independent processes operating in parallel-a normalization process that increases "different" RT as irrelevant disparity increases and a response-competition process that results in faster RT with corresponding increases in irrelevant disparity. What we are suggesting is that the effects of these two processes may have essentially cancelled out one another. While this appeal to coincidence should perhaps not be taken too seriously, it probably should not be dismissed as being totally impossible, either.

(Received for publication January 17, 1980; revision accepted May 9,1980 .) 